\title{
Analysis of proline accumulation, antioxidant capacity and HSP expression in mutant rice lines with different heat tolerance
}

\author{
Jian-Zhong Huang ${ }^{1,2}$, Shou-Ling $\mathrm{Xu}^{1}$, Ting-Chen $\mathrm{Ma}^{3}$, You-Fa $\mathrm{Li}^{4}$, $\mathrm{Hao}-\mathrm{Wei} \mathrm{Fu}^{4}, \mathrm{Ze}-\mathrm{Fu} \mathrm{Li}^{3}$, \\ Qing-Yao Shu ${ }^{2 *}$
}

\author{
${ }^{1}$ Institute of Nuclear Agricultural Sciences, Zhejiang University, Hangzhou 310058, China \\ ${ }^{2}$ National Key Laboratory of Rice Biology, Institute of Crop Sciences, Zhejiang University, \\ Hangzhou 310058, China \\ ${ }^{3}$ Rice Research Institute, Anhui Academy of Agricultural Sciences, Hefei 230031, China \\ ${ }^{4}$ Jiaxing Academy of Agricultural Sciences, Jiaxing314016, Zhejiang, China
}

\section{*Corresponding author: qyshu@zju.edu.cn}

\begin{abstract}
Three mutant rice (Oryza sativa L.) lines (AG1, AG2 and AG3) were selected as heat tolerant mutants from a gamma-ray-irradiated population of a heat-susceptible line (AG), based on their floret fertility grown under high temperatures. They were subjected to heat stress treatment $\left(45^{\circ} \mathrm{C}, 22 \mathrm{hrs}\right)$ at the 5-leaf stage, together with a heat-tolerant cultivar N22 and AG. Analysis of seedling root growth by WinRHIZO scanning revealed that N22 and AG3 were more heat-tolerant than the other lines (AG being the most heat susceptible). Following heat stress, a significantly higher level of oxidative damage, as indicated by TBARS, was observed in AG than in N22 and AG3. The proline accumulation was significantly higher in N22 and AG3 (12- to 13.5- fold) than AG (2.5- fold). Similarly, significantly greater increases of total antioxidant capacity (T-AOC) and superoxide dismutase (SOD) activity were observed in N22 and AG3 than in AG. The expression of four heat shock proteins was also investigated using qPCR: OsHSP16.9, OsHSP80.3 and OsHSP100.9 were induced by heat stress to various levels while OsHSP72.6was down-regulated in all tested lines. The heat-induced expression of OsHSP16.9 and OsHSP100.9 in N22 and AG3 was about twice that of AG. Higher proline accumulation and expression of the three HSP genes, as well as elevation ofT-AOC and SOD activity were observed in the heat-tolerant N22 and the mutant line AG3 under heat stress.
\end{abstract}

Keywords: heat-tolerance; rice; Oryza sativa; proline accumulation; antioxidant system; heat-shock protein. Abbreviations: HSPs_heat shock proteins; POD_peroxidase; SOD, superoxide dismutase; ROS, reactive oxygen species; T-AOC, total antioxidant capacity; TBARS, thiobarbituric acid reactive substances.

\section{Introduction}

Rice (Oryza sativa L.) is a staple food crop and a principal calorie source for Asian people (Khush, 2001). The optimal temperature for rice grain formation and yield is around $25^{\circ} \mathrm{C}$, and pollen viability and production begin to decline as daily maximum temperatures exceeds $33^{\circ} \mathrm{Cand}$ completely fail at $40^{\circ} \mathrm{C}$ (Luo, 2011); temperatures exceeding $35^{\circ} \mathrm{C}$ can cause a high percentage of spikelet sterility (Yoshida, 1981). The irreversible damage caused by high temperatures to seedling growth, leaf photosynthesis, and spikelet fertility contribute to yield reduction in rice. Under global warming scenarios, creating new rice cultivars (or germplasm for breeding) provides a sustainable solution to reduce the risk of high temperatures on world food security.

Overwhelming evidence indicates that heat stress is accompanied by increased levels of reactive oxygen species (ROS), which can cause oxidative damage to the plant cells. Antioxidant capacity of a plant, therefore, can be important in the tolerance of heat stress (Suzuki and Mittler, 2006; Kotak et al., 2007; Nouri and Komatsu, 2013). As an adaptive mechanism, plants grown under high temperature often accumulate osmo-protectants such as proline and soluble sugars (Wahid et al., 2007). Proline is believed to be beneficial to protect cellular structures from damage and maintain function under extreme temperatures (Bita and Gerats, 2013). In addition, cells from virtually all organisms respond to heat stress by the rapid synthesis of heat shock proteins (HSPs).
In higher plants, HSPs are induced under heat shock at any stage of development and major HSPs are highly conserved among distinct organisms (Vierling, 1991). HSPs belong to multigene families encoding molecular chaperones involved in various processes including maintenance of protein homeostasis as a requisite for optimal development and survival under stress conditions. These proteins play important roles in the development of thermotolerance and protection from cellular damage associated with stress in higher plants (Wahid et al., 2007). Over-expression of heat shock proteins in rice plants has been shown to increase thermotolerance (Murakami et al., 2004; Fragkostefanakis et al., 2015; Wang et al., 2015a).

In this study, we mutated a heat-susceptible line ( $\mathrm{Fu} 296)$ with gamma ray irradiation and identified three mutant lines (AG1, AG2 and AG3) with enhanced floret fertility when grown under high temperatures. To investigate the physiological and molecular basis of increased thermotolerance of the rice mutants, i.e., AG1, AG2 and AG3, we analyzed the levels and activities of antioxidants, oxidative stress (TBARS), proline accumulation and the expression of four heat-shock proteins under heat stress. The results were compared to plant performance in terms of shoot and root growth, especially seedling root growth which provided a good indicator of heat stress tolerance.

The results suggested that higher proline accumulation and HSP expression as well as the elevated T-AOC and SOD activity under heat stress may have contributed to the increased thermotolerance of the mutant lines. 


\section{Results}

\section{Plant growth under heat stress}

After heat treatment at $45^{\circ} \mathrm{C}$ and $60 \%$ humidity for 22 hours, slight but insignificant decrease in plant height was observed in N22 and all tested rice lines at the leaf 5 stage (Fig. 1a). However, root growth was more affected, with significantly reduced total root length $(-9 \%$, Fig. 1 b), reduced average root diameter $(-20 \%$, Fig. 1c) and reduced total root surface area (data not shown) in the relatively heat susceptible progenitor line AG, whereas the decreases in the heat-tolerant cultivar N22 and mutant line AG3 were insignificant (Fig. 1b, 1c). Significant declines were also observed in AG1 (-5\% and $-14 \%$ for total root length and root average diameter, respectively) (Fig. 1b, 1c) and in AG2 (-9\% of root average diameter) (Fig. 1c).

Changes in seedling root parameters under heat stress indicated that N22 and AG3 were more heat-tolerant than AG, AG1 and AG2, consistent with observations of their fertility performance in paddy field. However, heat-inhibited growth in the relatively heat susceptible line AG was indistinguishable from that of AG1, which showed improved heat-tolerance in terms of fertility. This was probably due to the insufficient time following heat stress for plants to manifest the difference, both in the case of shoot (Fig. 1a) and root growth (Fig. 1b, 1c).

\section{Oxidative damage in tice lines with different thermotolerance}

High levels of TBARS indicate high levels of oxidative damage and often the heat-susceptibility of plants (Larkindale et al., 2005). Consistent with this, the heat-tolerant cultivar N22 and the relatively heat-tolerant line AG3 had relatively lower levels (150-180\% of control) of TBARS following heat stress. By contrast, the heat-susceptible progenitor line AG had the highest level $(270 \%$ of control) of TBARS. TBARS levels of the moderate heat-tolerant lines AG2 and AG3 were also intermediate (210-220\% of control; Fig. 1d).

\section{Proline levels and antioxidant activities following heat stress}

Afterheat stress, proline content in leaves was significantly increased in all tested lines (Fig. 2a). The heat-induced proline increases in N22 and AG3 was about 12- to 13.5- fold, significantly higher than those of the three other less heattolerant lines (2.5- to 7.5) (Fig. 3a).

T-AOC levels were also increased significantly after heat stress. The heat-induced increase of T-AOC was much higher in N22 (2.4-fold) and AG3 (1.9-fold) than in the other three lines (1.3to 1.7 - fold) (Fig. 2 b).

Following heat stress, POD activity declined in the test lines except in the AG (Fig. 2c). However, the activity of SOD was significantly increased in N22 and AG3 exposed to heat stress and was significantly lower in the heat-susceptible line AG (Fig. 2d).

\section{Expression of four heat-shock protein genes}

To examine the heat-induced response of molecular chaperones, the expression of four HSP genes encoding, respectively, small HSP(OsHSP16.9), HSP70 (OsHSP72.6), HSP90(OsHSP80.3) and HSP100(OsHSP100.9), were analyzed (Table 1). Expression of the three of HSP genes, i.e., OsHSP16.9, OsHSP80.3and OsHSP100.9, was increased by 1.5- to 2.9-, 1.3- to 1.9- and 11to 22 - folds respectively, while that of OsHSP72.6 decreased by 1.1- to 1.7- folds following heat stress (Fig. 3).

The largest increase of OsHSP16.9 expression occurred in the heat tolerant rice cultivarN22 (about 2.9- fold), while the least in the heat-susceptible line AG (about 1.5-fold). The expression levels of OsHSP16.9 in the relatively heat-tolerant line AG3 and moderate tolerant lines AG1 and AG2 were increased by 2.5-, 2.1- and 2.4-fold, respectively (Fig. 3). The most dramatic increase was seen in the expression of OsHSP100.9with 22-fold increase in N22 and AG3, 15- to 17-fold in AG1 and AG2, and 11-fold in AG (Fig. 3).

\section{Discussion}

Global warming has been evident throughout the world in the past few decades with an increased frequency of occurrence of extreme temperatures in increasing land areas (Hansen et al., 2012). Development of rice cultivars which could withstand high temperatures will be achieved in part through understanding the molecular basis of heat stress responses in this crop.

At the cellular level, high temperatures can cause perturbations in membrane-linked processes due to increased fluidity of membrane lipids and alteration of permeability (Bischof et al., 1995). In addition, heat-induced protein denaturation and aggregation can cause enzyme inactivation. Membrane and protein damage lead to metabolic imbalances and the production of

toxic compounds and ROS (Wahid et al., 2007).

Compatible low molecular osmolytes such as proline and soluble sugars accumulate under heat stress (Wahid et al., 2007). Although Lv et al. (2011) found that growth of the prolineoverproduced Arabidopsis seedlings were inhibited during heat stress, other reports

showed that proline accumulation is necessary to regulate osmotic activities and protect cellular structures from increased temperatures by maintaining the cell water balance, membrane stability, and by buffering the cellular redox potential (Dobra et al., 2010; Bita and Gerats, 2013). Field trials with prolineoverexpressed soybean lines showed improved drought performance and higher heat tolerance compared to wild type cultivars (de Ronde et al., 2004; Verbruggen and Hermans, 2008). It is likely that the effects of proline accumulation in plants can be species- and organ- specific. This is in accordance with our finding in that the heat-tolerant rice cultivar N22 and the relatively heat-tolerant mutant line AG3 had the highest level of proline among the tested rice lines exposed to heat stress. Multiple signaling pathways are implicated in response to heatstress. Protection of plant from the oxidative damage by elevation of antioxidant activity in stressed tissues is an important component of a plant under heat stress (Kotak et al., 2007). In general, the T-AOC reflects the enzyme and non-enzyme antioxidant system as a whole. SOD among others is a major antioxidant enzyme in plants. They are responsible for the removal of free radicals such as $\mathrm{O}^{2 \cdot-}$, thus reducing the injury caused by lipid oxidation of cell membrane (Moller et al., 2007). The higher levels of T-AOC and SOD activity in N22 and AG3 can be important to their heat-tolerance. Although most HSPs are induced to be synthesized by heat stress, OsHSP genes exhibited very diverse expression patterns in different rice tissues (Vierling, 1991; Ye et al., 2012). Large variations exist in the literature with respect to the expression levels of specific OsHSP genes. For instance, while Ye et al. (2012) found thatLoc_Os11g47760 (HSP70 family) in seedling of rice cv. IRAT109 was upregulated 6 - fold under $42^{\circ} \mathrm{C}$ for $12 \mathrm{~h}$. Hu et al. (2009) found it downregulated in rice cv. ZH11. Similar contradictory results were obtained regarding the expression of LOC_Os01g08860 (sHSP family) under heat stress by different research groups. The expression of this gene was found to be up-regulated by heat treatment in rice seedlings from $>4$-fold (Hu et al., 2009), >8-fold (Jung and An, 2012) and even to >227fold (Ye et al., 2012). However, it was not detected under $25^{\circ} \mathrm{C}$, $37^{\circ} \mathrm{C}$ and $42{ }^{\circ} \mathrm{C}$ by Ouyang et al. (2009). Using proteomics, Jagadish et al. (2011) found that heat shock proteins were significantly upregulated under heat stress in the highly heattolerant cultivar N22 during rice anthesis. The high expression levels of OsHSP16.9, OsHSP80.3 and OsHSP100.9in N22 following heat stress were well correlated with the high heat tolerance of this rice variety. The expression levels of these three HSPs in the relatively heat tolerant mutant line AG3 were also upregulated to similar level as those of N22. On the other hand, the relatively heat-susceptible line AG exhibited the least increase in all these genes among the tested rice lines. 
Table 1. Primer sequences used for qPCR.

\begin{tabular}{|c|c|c|c|}
\hline Gene & $\begin{array}{l}\text { Family; predicted } \\
\text { MW* }\end{array}$ & Primer sequences & Size (bp) \\
\hline LOC_Os01g04370 & sHSP; 16.9 kDa & $\begin{array}{l}\text { qHSP1-F: AAGATAGAGCAACCATGTCG } \\
\text { qHSP1-R: CTTCACCTCCTCCTTCTTGA }\end{array}$ & 221 \\
\hline LOC_Os03g02260 & HSP70; $72.6 \mathrm{kD}$ & $\begin{array}{l}\text { qHSP2-F: GCTGACAACCAGACGCAAGT } \\
\text { qHSP2-R: CGATCTCGGAGGCAACCT }\end{array}$ & 409 \\
\hline $\mathrm{LOC}_{-} \mathrm{Os}_{\mathrm{S}} 4 \mathrm{~g} 01740$ & HSP90; 80.3 kD & $\begin{array}{l}\text { qHSP3-F: AGCAGTGAACTGAAATGGCG } \\
\text { qHSP3-R: GAGAGAGTCTTGGAGGGCTT }\end{array}$ & 268 \\
\hline LOC_Os05g44340 & HSP100; 100.9 kD & $\begin{array}{l}\text { qHSP4-F: GTCATGCAGGAGGTGAGGAG } \\
\text { qHSP4-R: TCGATGTACACCGTGCAGTT }\end{array}$ & 320 \\
\hline
\end{tabular}

*Abbreviations (using MW) for the four HSP genes are HSP16.9, HSP72.6, HSP80.3 and HSP100.9, respectively.

(a)

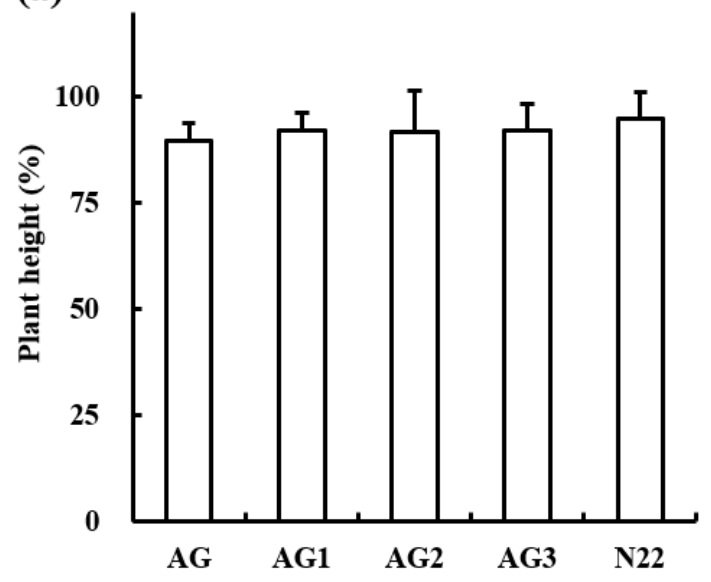

(c)

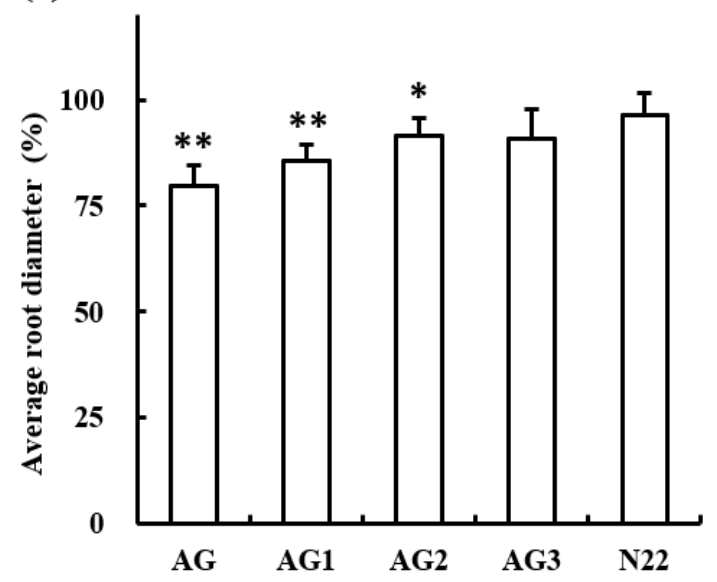

(b)

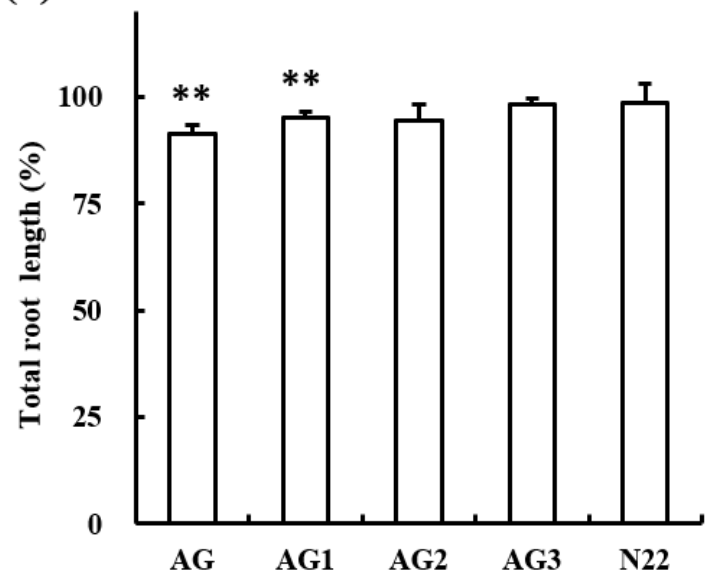

(d)

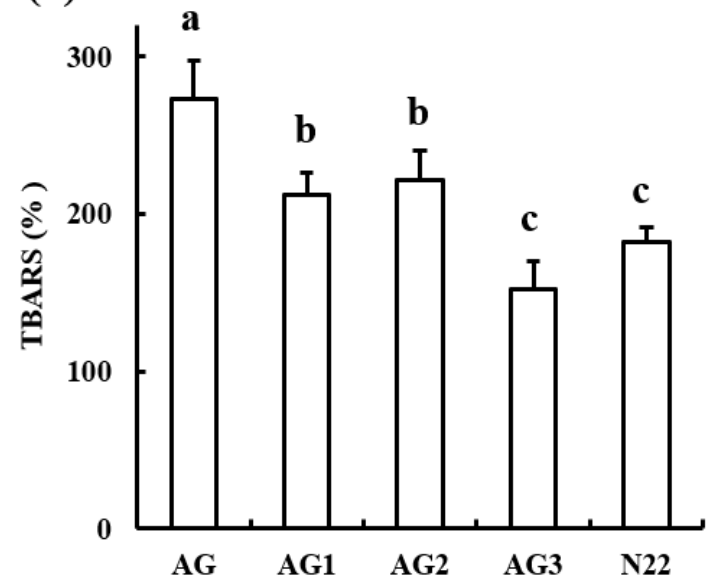

Fig 1. Effect of high temperature $\left(45^{\circ} \mathrm{C}, 22 \mathrm{hrs}\right)$ on the growth and oxidative damage in rice seedlings. Plant height (a), total root length (b) and average root diameter (c) were relative to those of the same linegrown without heat stress. (a) plant height; (b) total root length; (c) average root diameter; (d) TBARS levels. Root parameters were obtained using WinRhizo root image analysis system. (a, b, c) Bars with * indicate significant difference compared to respective control at $\mathrm{P} \leq 0.05$ and $* *$ at $\mathrm{P} \leq 0.001$; (d) Bars with the same lower-case letter are not significantly different at $\mathrm{P} \leq 0.05$. Statistical significance was determined by using the Student's t-test. Experiments were performed in triplicate, each with 12 seedlings per line harvested from the same container. 
(a)

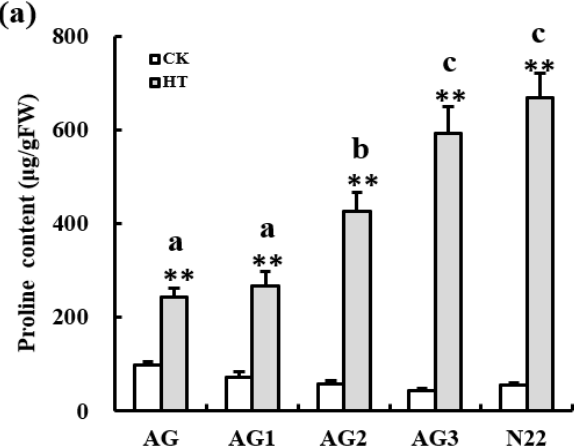

(c)

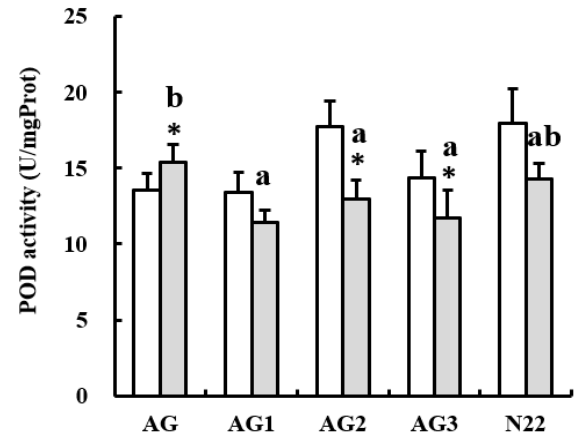

(b)

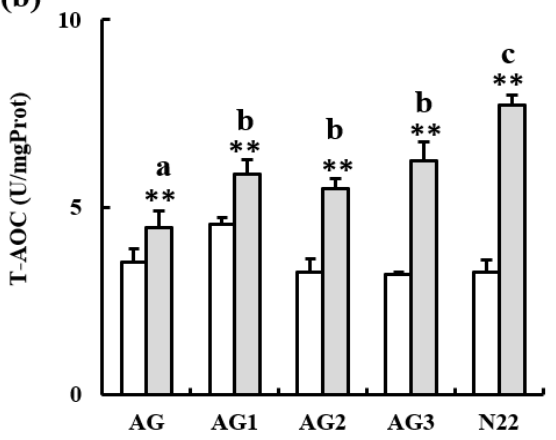

(d)

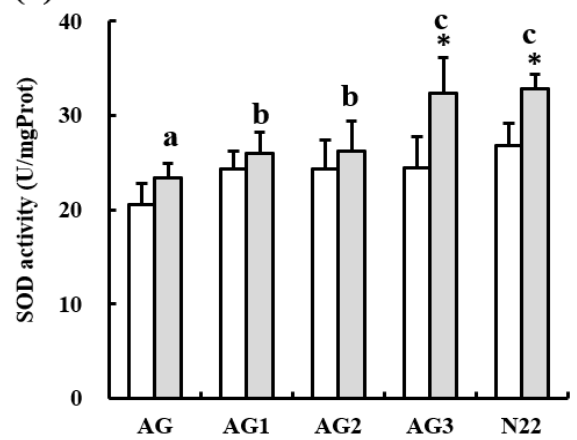

Fig 2. Leaf proline and T-AOC levels and activities of antioxidant enzymes (POD and SOD) in rice seedlings with different thermotolerance before (white bars) and after heat stress (grey bars). Bars with * indicate significant difference with respective control at $\mathrm{P} \leq 0.05$ and $* *$ at $\mathrm{P} \leq 0.001$. Bars with the same lower-case letter are not significantly different at $\mathrm{P} \leq 0.05$. Experiments were performed in triplicate, each with 12 seedlings per line harvested from the same container.

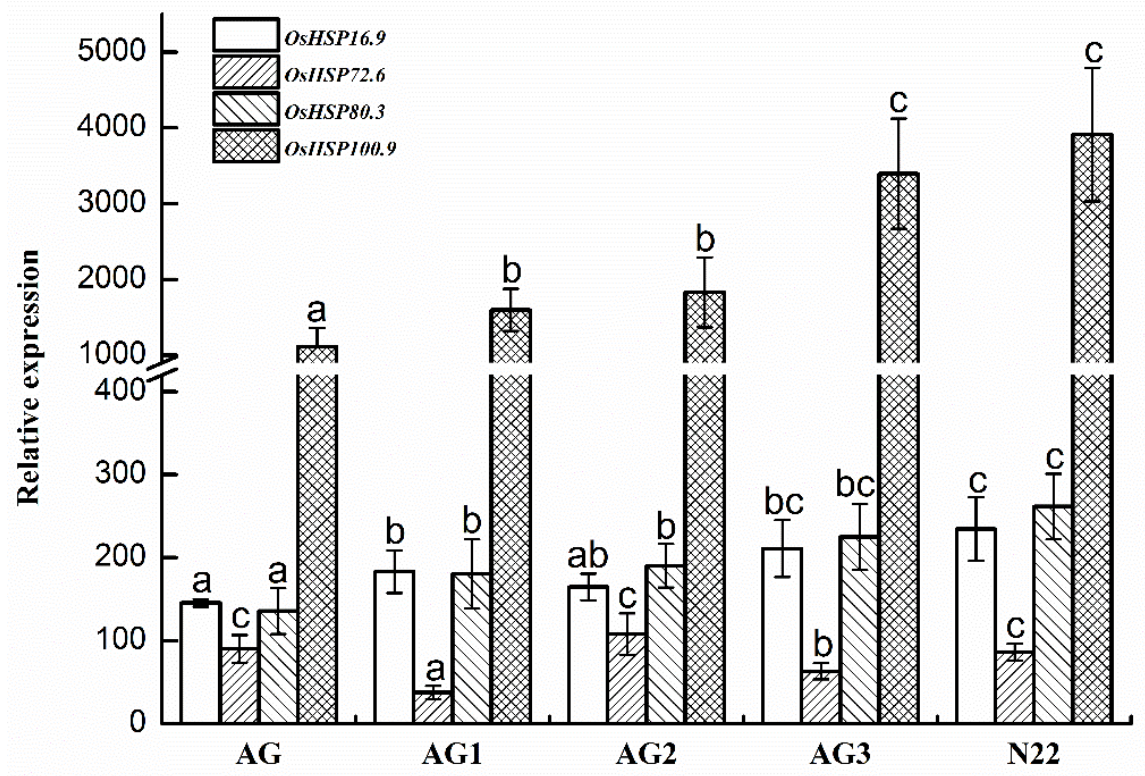

Fig 3. Expression of four HSP genes in rice seedlings with different thermotolerance following heat stress. Total RNA was extracted from rice leaf tissue at the 5-leaf stage from 12 seedlings per line harvested from the same container. The experiment was done in triplicates. Expression level of the progenitor parental line AG without heat stress was set as 100\%. A. OsHSP16.9(LOC_Os01g04370); B. OsHSP72.6 (LOC_Os03g02260); C. OsHSP80.3 (LOC_Os04g01740); D. OsHSP100.9 (LOC_Os05g44340). Bars with * indicate significant difference with control at $\mathrm{P} \leq 0.05$ and ${ }^{* *}$ at $\mathrm{P} \leq 0.001$. Bars with the same lower-case letter are not significantly different at $\mathrm{P} \leq 0.05$. 
The ability to withstand and to acclimate to supra-optimal temperatures can involve numerous traits at morphological, physiological and molecular levels. Development and maintenance of thermotolerance in plants will have to do with the antioxidant activity, membrane lipid unsaturation, gene expression and translation, protein stability, and accumulation of compatible solutes (Larkindale et al., 2005; Bita and Gerats, 2013). Constitutive elevation of osmolytes and antioxidants seems to be happening under non-stress conditions, which is potentially useful to plant growth; Although an episode of high temperature at an earlier developmental stage may not directly affect the heat response of a subsequent stage (Wollenweber et al., 2003), it is likely that the response of the osmolyte and antioxidant mechanisms at seedling stage happens at later growth as well, although not examined in the current experiments. Therefore, overall elevation of antioxidant capacity and membrane stabilization and protein protection by compatible osmolytes and molecular chaperones are certainly beneficial to both vegetative and reproductive stages in amelioration of heatinduced injury in plants.

\section{Materials and methods}

\section{Plant materials}

For generation of heat tolerant mutants, rice seeds of cv. Fu 296 were irradiated with $\gamma$ rays (350 Gy) and their progenies were subjected to screening for improved heat tolerance from $\mathrm{M}_{2}$ to $\mathrm{M}_{4}$ in paddy fields in the summers of 2012 to 2015 in Hangzhou, China $\left(30.42^{\circ} \mathrm{N}, 120.12^{\circ} \mathrm{E}\right)$. Fu 296 is an early inbred indica rice cultivar, developed through the pedigree breeding from $\gamma$ rayirradiation of $\mathrm{F}_{1}$ dry seeds Z96-03 $\times$ Guangchangzhan. Improved traits include: erect leaves, sturdy stems, large panicle and a higher filled seed-set, higher and stable grain yield. It is about $95 \mathrm{~cm}$ of height and requires $\sim 111 \mathrm{~d}$ from sowing to harvest. The cultivar was registered in Hunan Province in China in 2008 (Liu et al., 2010). Only mutant lines showing the same phenotype with the cultivar's characteristic traits were selected from the $\gamma$-irradiated progenies of Fu296. A number of mutant lines showing improved heat tolerance were selected at anthesis with increased fertilities at natural high temperatures. We selected three mutant lines (AG1, AG2 and AG3) with different thermotolerance for the following experiment: AG3 was more tolerant than AG1 and AG2, which were in turn more tolerant than the progenitor parental line Fu296 (hereafter AG) (Supplemental Table 1).

\section{Heat treatment}

Rice seedlings $\left(\mathrm{M}_{5}\right)$ consisting of three mutant lines and their progenitor, and a reference heat-tolerant cultivar (N22; Yoshida et al., 1981; Jagadish et al., 2011), were raised in soil culture in plastic containers (Wang et al., 2009). Twelve plants per line and four lines each container was grown in a container (length/wide/depth: 60/40/25cm) with water logged paddy soil. Containers were maintained at field saturation capacity and irrigation was done when required. At 5-leaf stage, part of onemonth old seedlings was subjected to heat stress by incubation in controlled growth chambers at $45^{\circ} \mathrm{C}$ and $60 \%$ humidity for 22 hours and a $16 \mathrm{~h}$ photoperiod under $160 \square \mathrm{mol} \mathrm{m}^{-2} \mathrm{~s}^{-1}$ light. The remaining seedlings of each line or cultivar were kept at $\sim 30^{\circ} \mathrm{C}$ as controls. Upon the completion of stress treatment, the fifth leaf of each plant was cut for immediate assays of antioxidants, or frozen in liquid nitrogen and stored under $-70^{\circ} \mathrm{C}$ until required for further investigation. Experiments were performed in triplicate, each with 12 seedlings per line harvested from the same container.

\section{Plant growth measurement}

Before and immediately following heat treatment, six plants from each rice line were uprooted carefully from the soil. Plant height was measured, and seedling roots taken by cutting off the culms. Root samples were then washed carefully on a $0.5-\mathrm{mm}$ mesh screen. After removal of debris, roots were spread out on a glass plate and scanned using the root scanner WinRHIZO system (WinRHIZO, Regent Instrument, Montreal, QC, Canada). Total root length, total root surface area and average root diameter were then obtained.

\section{Proline, TBARS and antioxidants assay}

Leaf samples were homogenized using a chilled mortar and pestle with ice-cold extraction buffer supplied with the kits (Nanjing Jiancheng Bioengineering Institute, Jiangsu, China). Homogenate was centrifuged at $10,000 \times g$ for $10 \mathrm{~min}$ at $4^{\circ} \mathrm{C}$. The supernatant was used for measurement of thiobarbituric acid reactive substances (TBARS), proline, total antioxidant capacity (T-AOC), soluble protein, peroxidase and superoxide dismutase (SOD) using corresponding assay kits. All reagents used were provided with the supplier's kits.

\section{Heat-shock protein expression}

For quantitative PCR (qPCR) analysis, total RNA was extracted from rice leaf tissue at the 5-leaf stage using the Qiagen Spin Plant RNA Mini Kit (Qiagen, Germany). cDNAs were reverse transcribed from $100 \mathrm{ng}$ total RNA using the oligo-d $\mathrm{T}_{18}$ primer and GoScript ${ }^{\mathrm{TM}}$ Reverse Transcription System Kit (Promega, USA) according to the manufacturer's instructions. qPCRs were performed using a SYBR Green GoTaq ${ }^{\circledR}$ qPCR Master Mix kit (Promega, USA). One primer pair each was designed for qPCR analysis of the fourHSPgenes (qHSP1-4, Table 1). All qPCRs were performed in $20 \mu \mathrm{L}$ volumes with $1 \mu \mathrm{L}$ cDNA in $1 \times$ GoTaq ${ }^{\circledR}$ qPCR Master Mix (containing PCR buffer, $\mathrm{MgCl}_{2}$, dNTPs, Taq DNA polymerase), and $0.4 \mu \mathrm{M}$ of each primer. The following programme was used for all qPCRs: $10 \mathrm{~min}$ at $95^{\circ} \mathrm{C}$, 40 cycles of $30 \mathrm{~s}$ at $95^{\circ} \mathrm{C}, 30 \mathrm{~s}$ at $55^{\circ} \mathrm{C}$, and $60 \mathrm{~s}$ at $72{ }^{\circ} \mathrm{C}$. The rice ubiquitin gene was used as an internal reference (primersUbq-F, 5'-GCTCCGTGGCGGTATCAT-3'; Ubq-R, 5'CGGCAGTTGACAGCCCTAG-3'; Wang et al., 2015b). Relative gene expression was calculated using the $2^{-\Delta \Delta C t_{m}}$ method (Livak and Schmittgen, 2001). qPCRs were repeated with three biological replicates, each with RNA extracted from two individual seedlings.

\section{Conclusions}

Heat stress treatment at the 5-leaf stage demonstrated that the mutant line AG3 had increased heat tolerance compared with its heat-susceptible parental line $\mathrm{Fu}$ 296. Higher proline accumulation and expression of OsHSP16.9 and OsHSP100.9, as well as elevated T-AOC and SOD activity might constitute part of the mechanisms that underpin its increased thermotolerance of AG3. The mutant line could be used for breeding heat tolerant rice cultivars in future breeding programmes.

\section{Acknowledgements}

This work was supported by the FAO/IAEA Coordinated Research Project CRP 23029, Research Contract No16818.

\section{References}

Ahloowalia BS, Maluszynski M (2001) Induced mutations - a new paradigm in plant breeding. Euphytica. 118:167-173.

Bischof JC, Padanilam J, Holmes WH, et al (1995) Dynamics of cell membrane permeability changes at supraphysiological temperatures. Biophys J. 68: 2608-2614.

Bita CE, Gerats T (2013) Plant tolerance to high temperature in a changing environment: scientific fundamentals and production of heat stress-tolerant crops. Front Plant Sci. 4: 273. 
de RondeJA, Laurie RN, Caetano T, et al (2004) Comparative study between transgenic and non-transgenic soybean lines proved transgenic lines to be more drought tolerant. Euphytica. 138: 123-132.

Dobra J, Motyka V, Dobrev P, et al (2010) Comparison of hormonal responses to heat, drought and combined stress in tobacco plants with elevated proline content. Plant Physiol. 167: 1360-1370.

Fragkostefanakis S, Röth S, Schleiff E, Scharf KD (2015) Prospects of engineering thermotolerance in crops through modulation of heat stress transcription factor and heat shock protein networks. Plant Cell Environ.38: 1881-1895.

Hansen J, Sato M, Ruedy R (2012) Perception of climate change. P Natl Acad Sci USA.109: E2415-E2423.

Hu W, Hu G, Han B (2009) Genome-wide survey and expression profiling of heat shock proteins and heat shock factors revealed overlapped and stress specific response under abiotic stresses in rice. Plant Sci.176: 583-590.

Jagadish SVK, Muthurajan R, Rang ZW, et al (2011) Spikelet proteomic response to combined water deficit and heat stress in rice (Oryza sativa cv. N22). Rice.4: 1-11.

Jung KH, An G (2012) Application of MapMan and RiceNet drives systematic analyses of the early heat stress transcriptome in rice seedlings. Plant Biol.55: 436-449.

Khush GS (2001) Green revolution: the way forward. Nat Rev Genet.2: 815-822

Kotak S, Larkindale J, Lee U, et al (2007) Complexity of the heat stress response in plants. Curr Opin Plant Biol.10: 310-316.

Larkindale J, Hall JD, Knight MR, Vierling E (2005) Heat stress phenotypes of Arabidopsis mutants implicate multiple signaling pathways in the acquisition of thermotolerance. Plant Physiol.138: 882-897.

Liu Q, Yan W, Yu F, Qi Y, Wu G, Jin W, Jin Q (2010) Breeding of 'Fu 296'rice with high yield and good quality by ${ }^{60} \mathrm{Co} \gamma$-rays irradiaton dry seeds of hybrid F1. J Nucl Agr Sci. 24: 744-747.

Livak KJ, Schmittgen TD (2001) Analysis of relative gene expression data using real-time quantitative PCR and the $2^{-\triangle \Delta C T}$ method. Methods. 25: 402-408.

Luo Q (2011) Temperature thresholds and crop production: a review. Climatic Change.109: 583-598.

Lv WT, Lin B, Zhang M, Hua XJ (2011) Proline accumulation is inhibitory to Arabidopsis seedlings during heat stress. Plant Physiol.156: 1921-1933.
Moller IM, Jensen PE, Hansson A (2007) Oxidative modifications to cellular components in plants. Annu Rev Plant Biol.58: 459481.

Murakami T, Matsuba S, Funatsuki H, et al (2004) Over-expression of a small heat shock protein, sHSP17.7, confers both heat tolerance and UV-B resistance to rice plants. Mol Breeding.13: 165-175.

Nouri MZ, Komatsu S (2013) Subcellular protein overexpression to develop abiotic stress tolerant plants. Front Plant Sci.4: 2.

Ouyang Y, Chen J, Xie W, Wang L, Zhang Q (2009) Comprehensive sequence and expression profile analysis of Hsp20 gene family in rice. Plant Mol Biol.70: 341-357.

Suzuki N, Mittler R (2006) Reactive oxygen species and temperature stresses: a delicate balance between signaling and destruction. Physiol Plant. 126: 45-51.

Verbruggen N, Hermans C (2008) Proline accumulation in plants: a review. Amino Acids.35: 753-759.

Vierling E (1991) The roles of heat-shock proteins in plants. Annu Rev Plant Physiol Plant Mol Biol.42: 579-620.

Wahid A, Gelani S, Ashraf M, Foolad MR (2007) Heat tolerance in plants: an overview. Environ Exp Bot.61: 199-223.

Wang A, Yu X, Mao Y, et al(2015a) Overexpression of a small heatshock-protein gene enhances tolerance to abiotic stresses in rice. Plant Breeding.134: 384-393.

Wang C, Zhang X, Fan Y, et al(2015b) XA23 is an executor R protein and confers broad-spectrum disease resistance in rice. Mol Plant.8: 290-302.

Wang H, Huang J, Ye Q, Wu D, Chen Z (2009) Modified accumulation of selected heavy metals in $\mathrm{Bt}$ transgenic rice. $\mathrm{J}$ Environ Sci.21: 1607-1612.

Wollenweber B, Porter JR, Schellberg J (2003) Lack of interaction between extreme high-temperature events at vegetative and reproductive growth stages in wheat. J Agron Crop Sci. 189: 142150 .

Ye S, Yu S, Shu L, Wu J, Wu A, Luo L (2012) Expression profile analysis of 9 heat shock protein genes throughout the life cycle and under abiotic stress in rice. Chin Sci Bull.57: 336-343.

Yoshida S (1981) Fundamentals of rice crop science. IRRI, Los Baños, 269.

Yoshida S, Satake T, Mackill DS (1981) High temperature stress in rice. IRRI Res Pap Ser 67. 